\title{
ANÁLISE DA ECONOMICIDADE DE CENÁRIO PARA REATIVAÇÃO DE CAMPO MADURO DE PETRÓLEO: UM ESTUDO DE CASO REAL
}

\section{ANALYSIS OF THE SCENARIO ECONOMICS FOR THE REACTIVATION OF OIL MATURE FIELD: A CASE STUDY}

\section{J. C. M. MENDONÇA ${ }^{1, *}$, A. L. M. $\operatorname{COSTA}^{2}$ e J. E. NEVES ${ }^{2}$}

${ }^{1}$ Universidade Federal do Rio de Janeiro, Programa de Engenharia Civil, Rio de Janeiro, Rio de Janeiro, Brasil

${ }^{2}$ Universidade Federal de Campina Grande, Unidade Acadêmica de Engenharia de Petróleo, Campina Grande, Paraíba, Brasil

*Autor correspondente. Universidade Federal do Rio de Janeiro, Programa de Engenharia Civil, Rio de Janeiro, Rio de Janeiro, Brasil, Telefone: +55 21

997765369

Endereço de e-mail: jcarlosmmendonca@gmail.com (J. C. M. Mendonça).

\section{A R T I C L E I N F O}

Article history:

Received 2018-10-13

Accepted 2018-12-21

Available online 2019-04-25

palavras-chave

Campos maduros de petróleo

Análise econômica

Fluxo de caixa descontado

keywords

Oil mature field

Economic analysis

Discounted cash flow

\section{R E S U M O}

Como um novo rumo da indústria petrolífera, a reativação de campos maduros surge no Brasil com as rodadas de licitação de campos com acumulações marginais da ANP. Este trabalho tem como objetivo analisar tecnicamente dados reais de um campo maduro de petróleo através de cenário próprio para a revitalização e verificar se há uma viabilidade, utilizando de indicadores econômicos, para oferta durante o leilão do campo para a consequente continuação da produção nesse novo empreendimento. A análise econômica se deu pelo método do fluxo de caixa descontado e cálculo dos indicadores VPL e o TIR, os quais apontam a viabilidade econômica e o retorno do projeto. Neste estudo, foram encontrados ótimos valores destes indicadores para este campo, o que aponta um bom projeto para tal ação técnica, resultando em ótimos retornos de renda e, consequentemente, uma oportunidade para a empresa investir.

A B S T R A C T

As a new direction for the oil industry, the reactivation of mature fields appears in Brazil with the ANP bidding rounds of fields with marginal accumulations. This work aims to analyze technically real data from a mature oil field through a scenario for revitalization and verify if there is a feasibility, using economic indicators, to offer during the field bid for the consequent continuation of production in this new venture. The economic analysis was based on the discounted cash flow method and the calculation of the NPV and the IRR indicators, which indicate the economic viability and the return of the project. In this study, good values of these indicators were found for this field, which indicates a good project for such technical action, resulting in excellent returns of income and, consequently, an opportunity for the company to invest. 


\section{INTRODUÇ̃̃̃O}

Grandes descobertas petrolíferas estão cada vez mais escassas e a maioria das reservas mundiais está em reservatórios maduros como afirmados por Soliman et al. (1999), "a constatação de que a maioria das reservas mundiais está em reservatórios maduros resulta na urgência em desenvolver métodos para resolver a produção excessiva de água e o aumento da eficiência na recuperação".

Quanto aos campos dos primórdios da indústria petrolífera brasileira, estes encontram-se hoje em avançada maturidade e enquadram-se em um de dois cenários reais: a recorrência pelas grandes empresas aos altos investimentos em tecnologia para continuar produzindo estes campos ou a entrega destes por essas companhias à ANP devido ao desinteresse econômico inerente da situação.

As atividades onshore no Brasil somente se perpetuaram até os dias de hoje porque a maior parte dos investimentos foi herdada do passado, quando os esforços exploratórios eram primariamente feitos nesses campos, os quais, atualmente, continuam sua vida produtiva com a extração de barris marginais de reservatórios já exauridos. Como, quando se fala em atividades onshore no Brasil, consideram-se aquelas bacias mais maduras, deve-se resgatar que essas bacias costumam situar-se naqueles locais onde nasceu a indústria petrolífera nacional, onde há uma demanda social que compele o esforço por parte da indústria a continuar o investimento, pois todas essas áreas são ainda hoje marcadas pela pobreza e baixo dinamismo econômico (Senna, 2011).

Segundo Monteiro (2009), um estudo detalhado realizado pela ANP, em dezembro de 2005 , tendo em vista a Licitação de Campos Marginais constatou que dos 217 campos da PETROBRAS, situados em bacias terrestres, 157 deles se encontravam na categoria conhecida como campos marginais, apresentado reservas provadas inferiores a 530 mil barris de petróleo equivalente por campo. A ANP considera que a revitalização de tais campos não terá nenhum impacto significativo no desempenho econômico da PETROBRAS, concessionária de todos eles, porém de suma importância para a atividade econômica local.

É importante ressaltar que a revitalização dos campos maduros e marginais pode gerar grandes benefícios no âmbito socioeconômico para as regiões próximas às suas localizações, desde que esta pode contribuir para o desenvolvimento de áreas carentes, através da geração de empregos e oportunidades de serviço, da reativação do comércio e da indústria local.

Estes campos que não apresentam características econômicas favoráveis para as grandes produtoras podem ser viabilizados por companhias independentes, pois possuem necessidades menores de rentabilidade e custos indiretos menores. A modernização e revitalização dessas atividades exigem investimentos e tecnologias específicas, mas de menor magnitude, sendo, portanto, apropriado para pequenas $\mathrm{e}$ médias empresas. Apesar dos vários benefícios mostrados, a viabilidade econômica deste tipo de campo é muito dependente do preço do óleo ao longo da vida produtiva e de seu volume produzido. Logo, devem ser realizados estudos dessa viabilidade utilizando as técnicas apropriadas e os custos e rendas envolvidos nesse processo para que a decisão da revitalização ou não do campo seja propriamente tomada.

Diversos estudos já foram feitos na área de revitalização de campos maduros de forma mais geral ou específica de um determinado local, dando assim embasamento ao estudo de caso apresentado neste trabalho. Alguns exemplos de trabalhos são de: Junior \& Correia (2005) e Castiñeira (2008), que apresentaram abordagens mais gerais que levaram a uma proposta de regulação e a uma proposta técnica de ação nesses campos, respectivamente. Outros exemplos mais específicos são os trabalhos de: Narváez \& Ramisa (2005), Carneiro (2007) e Batista (2016), os quais falam das especificidades, riscos e dificuldades, avaliando economicamente a possibilidade de revitalizar campos maduros das regiões do Recôncavo Baiano, Norte do México e Potiguar, respectivamente.

Assim, este estudo, realizado na Universidade Federal de Campina Grande como trabalho de conclusão de curso para obtenção de título de bacharel em engenharia de petróleo, tem como objetivo analisar tecnicamente dados reais de um campo maduro de petróleo através de cenário próprio para a revitalização e verificar se há uma viabilidade, utilizando de indicadores econômicos, para oferta durante o leilão do campo para a consequente continuação da produção nesse novo empreendimento.

\section{METODOLOGIA}

Como metodologia aplicada neste trabalho, foram feitas análises econômicas por meio dos indicadores VPL e TIR de um projeto de reativação de campo maduro. Foram consideradas as seguintes premissas para o estudo:

1.0 campo em questão (campo real com nome e Empresa omitidos neste trabalho por questões de confidencialidade) encontra-se em estado de maturação, sendo necessária a avaliação econômica da viabilidade da retomada de produção;

2.Testes de produção indicaram reservatório limitado e depleção acentuada, com petróleo de elevado ${ }^{\circ}$ API e baixa viscosidade;

3.De acordo com os dados do campo, este possui volume original provado de $29000 \mathrm{~m}^{3}$ de óleo, tendo sido produzido apenas $2530 \mathrm{~m}^{3}$ (8,7\% do volume original) por meio do mecanismo de gás em solução;

4.Na situação atual do campo, existem dois poços, dos quais apenas um poço é passível de ser aproveitado, desde que o outro se encontra arrasado;

5.O poço aproveitável em questão foi fraturado e bons resultados foram obtidos, porém teve posteriores problemas no bombeio mecânico devido a produção de areia, provavelmente devido ao fechamento da fratura;

6.Este campo será reativado por meio da utilização do método de estimulação do fraturamento hidráulico;

7.Serão analisados os dados para uma retomada de produção por 5 anos.

Primeiramente, foram obtidos os dados sobre o campo para realização das análises. Dos poucos dados informados foi possível tirar concluir que o fraturamento feito no primeiro período de produção deste campo obteve bons resultados e que 
uma restauração por meio de refraturamento poderia ser uma boa proposta de ação de revitalização deste campo. Com esta proposta técnica em mente, partiu-se para a análise econômica desta. Então, foi calculado o fluxo de caixa do projeto a partir das diferenças entre os valores estimados de entrada e saída durante a vida útil deste projeto. Com este fluxo de caixa e a taxa de desconto adotada pela empresa em mãos, foi possível realizar os cálculos de VPL e TIR com a ajuda de funções disponíveis no software Microsoft Excel®, o qual possui funções próprias para o cálculo de tais indicadores.

A curva de produção estimada para o refraturamento, mostrada na Figura 1, foi obtida a partir da curva real do primeiro fraturamento feito no campo. A previsão de comportamento foi obtida adotando-se um declínio do tipo exponencial.

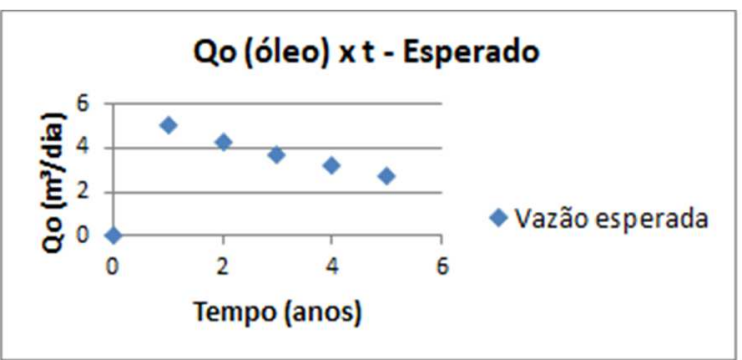

Figura 1 - Curva de produção estimada.

As análises técnica e econômica foram realizadas seguindo custos estimados em função de tabelas disponibilizadas pela ANP e receitas foram provenientes de curva de produção estimada. Com estas análises, será possível propor ação técnica para revitalização do campo e sugestão de oferta durante o leilão do campo.

\subsection{Cenário analisado para reativação do campo}

O campo em questão, atualmente fechado, será reativado por meio do refraturamento de um poço e posteriormente equipado para bombeio mecânico.

Alguns pressupostos técnicos e econômicos, com base na legislação vigente, deste cenário que servirão de base para o estudo são mostrados na Tabela 1 .

Tabela 1 - Pressupostos técnicos e econômicos do cenário em análise.

\begin{tabular}{cc} 
Data de concepção do & Agosto do ano 0 \\
estudo & Ano 5 \\
Horizonte do projeto & Ano 0 \\
Início do projeto & Janeiro do ano 1 \\
Início da produção & 10,0 \\
\hline Taxa mínima de & 3,13 \\
atratividade (\% a.a.) & \\
Taxa de câmbio empresarial & \\
$(\mathrm{R} \$ / \mathrm{U} \$)$ & 46,96 \\
Retenção de área & \\
$\left(\mathrm{U} \$ / \mathrm{km}^{2} / \mathrm{ano}\right)$ &
\end{tabular}

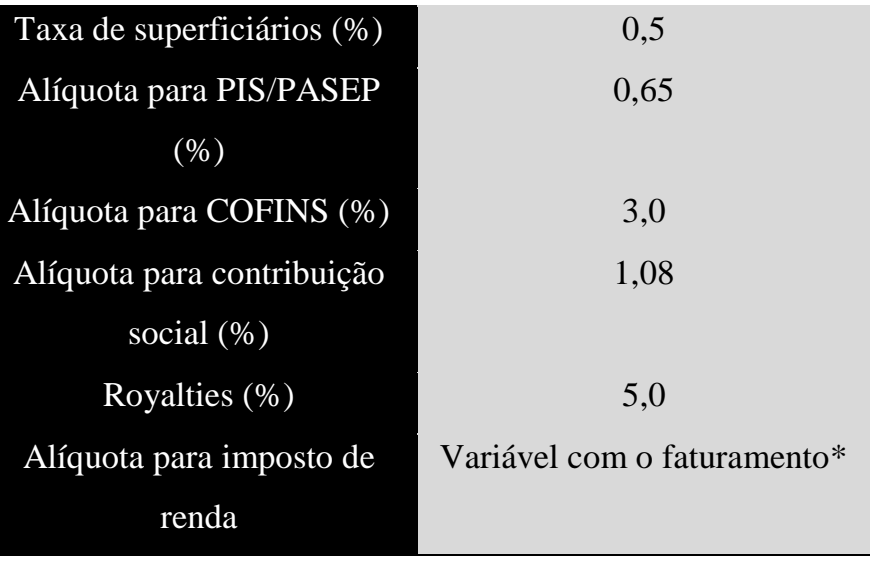

*A alíquota para o imposto de renda será calculada em cima do lucro real obtido, tendo uma porcentagem fixa de $15 \%$ com acréscimo de $10 \%$ se o lucro exceder o valor de $\mathrm{R} \$ 20.000,00$ por mês no período de aferição.

O fluxo de caixa deste estudo será baseado nas entradas provenientes da produção do óleo e nas saídas (sinalizadas por valores negativos) provenientes do investimento inicial, dos custos operacionais e dos tributos. Estes valores obtidos por projeção durante o tempo estimado para a análise são mostrados em tópicos a seguir.

\section{- Investimento inicial}

Este valor mostrado na Tabela 2 é proveniente do investimento para o fraturamento do poço produtor e instalação de equipamento de elevação.

Tabela 2 - Investimento inicial no cenário de reativação do campo maduro.

\begin{tabular}{cc} 
Ano & 0 \\
Total (MMU\$) & $-0,173913$ \\
\hline
\end{tabular}

- Receita bruta

Tabela 3. Receita bruta obtida no cenário de reativação do campo maduro.

\begin{tabular}{cccccc|c|} 
Ano & 0 & 1 & 2 & 3 & 4 & 5 \\
\hline Total & 0,0 & 0,597 & 0,5139 & 0,4423 & 0,3807 & 0,3276 \\
(MM & & 084 & 15 & 31 & 18 & 87 \\
U\$) & & & & & & \\
\hline
\end{tabular}

Estes valores mostrados na Tabela 3 são provenientes da receita gerada pela produção do óleo, com o preço do petróleo fixado em 52,02 U\$/BBL. O preço do gás natural possivelmente produzido não foi considerado nesta análise econômica, desde que a sua produção não é quantitativamente viável para a comercialização ou reinjeção no reservatório.

No ano 0 foi considerada a realização do estudo, o leilão do campo e o início do projeto com o refraturamento e seu tempo de execução, logo neste ano não houve produção, iniciando-se no ano 1.

\section{- Custos operacionais}

Os valores mostrados na Tabela 4 são provenientes dos 
custos operacionais fixos e variáveis envolvidos na produção do óleo. Dentre esses custos, figura os custos pela retenção da área do campo, a taxa paga aos superficiários e os custos de pessoal e de tratamento e escoamento do óleo.

No ano zero houve apenas custos com a retenção da área do campo e custos de pessoal.

- Tributos

Os valores mostrados na Tabela 5 são provenientes dos tributos vigentes em Lei que devem ser pagos à União pela produção de óleo e sua respectiva renda gerada. Os tributos considerados nos valores apresentados são: PIS/PASEP, COFINS, contribuição social, imposto de renda e royalties.

Não foram considerados tributos no ano zero devido à ausência de produção de petróleo, já que estes descontos são feitos em cima do volume produzido.

Tabela 4 - Custos operacionais envolvidos no cenário de reativação do campo maduro.

\begin{tabular}{ccccccc} 
Ano & 0 & 1 & 2 & 3 & 4 & 5 \\
Total (MMU\$) & $-0,006328$ & 0,597084 & 0,513915 & 0,442331 & 0,380718 & 0,327687 \\
\hline
\end{tabular}

Tabela 5 - Tributos advindos da produção de óleo no cenário de reativação do campo maduro.

\begin{tabular}{cccc|cc|c|}
\hline Ano & 0 & 1 & 2 & 3 & 4 & 5 \\
\hline Total (MMU\$) & 0,0 & $-0,167735$ & $-0,144371$ & $-0,124261$ & $-0,106952$ & $-0,092055$ \\
\hline
\end{tabular}

\section{RESULTADOS E DISCUSSÃO}

Foram determinados com os valores apresentados de investimento inicial, receita bruta, custos operacionais e tributos os fluxos de caixa líquido e acumulado, e estes se encontram listados pelo período de vida útil do projeto na Tabela 6 .

Em posse destes valores de fluxo de caixa, da taxa mínima de atratividade (a qual é de $10 \%$ ao ano) e por meio das funções próprias para o cálculo dos indicadores econômicos no software Microsoft Excel®, foi possível determinar os índices utilizados, VPL e TIR, para a análise econômica. Os valores destes índices do projeto em análise estão mostrados na Tabela 7.

Os valores encontrados foram extremamente satisfatórios, desde que o VPL foi maior que 0 (zero), o que representa um valor maior de entradas em relação às saídas do
Tabela 7 - Indicadores econômicos do projeto de reativação do campo maduro.

\begin{tabular}{l|c|c}
\hline Indicadores & VPL (U\$) & $\$ 719.222,01$ \\
Econômicos & TIR (\% a. a.) & $168 \%$ \\
\hline
\end{tabular}

projeto com uma diferença que gerará um possível lucro de mais de U\$700.000,00, enquanto o TIR mostrou-se bem maior que a taxa mínima de atratividade empregada, o que pode ser explicado pelos valores bem inferiores de saída em relação aos de entrada, assim representando um ótimo retorno do investimento. Estes valores indicam uma grande probabilidade do investimento ser altamente viável economicamente, o que pode ser entendido pela empresa como um aval para que esta se organize a fim de ofertar durante o leilão do campo.

Tabela 6 - Fluxos de caixa líquido (FCL) e acumulado (FCA) estimados para o projeto de reativação do campo maduro.

\begin{tabular}{cccccc|c}
\hline Ano & 0 & 1 & 2 & 3 & 4 & 5 \\
\hline FCL (MMU\$) & $-0,180241$ & 0,328917 & 0,283101 & 0,243667 & 0,209726 & 0,180513 \\
FCA (MMU\$) & $-0,180241$ & 0,148675 & 0,431776 & 0,675444 & 0,885171 & 1,065685 \\
\hline
\end{tabular}

\section{CONCLUSÕES}

Este trabalho tem caráter técnico e mostra a viabilidade de um cenário de reativação de campo maduro, campos estes que estão crescendo no cenário da indústria petrolífera, desde que grande parte dos campos no mundo já é classificada como tal.

O campo em estudo apresentou bons indicadores econômicos, o que demonstra um bom retorno do projeto e uma oportunidade em aberto para as empresas investirem e lucrarem. O indicador VPL mostrou um ótimo fluxo de caixa de entrada, o que significa lucro para a empresa, enquanto o indicador TIR mostrou uma alta probabilidade de $\mathrm{o}$ empreendimento ser viável economicamente. Assim, os dois indicadores se completam para obter-se uma análise econômica mais confiável e concisa.

Com base em estudos similares ao desenvolvido neste trabalho, as empresas se preparam para apresentação de ofertas em leilões de campos petrolíferos maduros.

\section{R E F E R E N C I A S}

BATISTA, P. B. M. Barreiras econômicas na exploração em terra de campos maduros e marginais: o caso da bacia Potiguar. 2016. Dissertação (Mestrado em Energia) Universidade de São Paulo, São Paulo, SP, 2016.

CARNEIRO, J. T. G. Avaliação geo-econômica de campos marginais oferecidos pela ANP na bacia do Recôncavo, Bahia, na sétima rodada de licitações. 2007. Dissertação 
(Mestrado em Ciências em Engenharia Civil) Universidade Federal do Rio de Janeiro, Rio de Janeiro, RJ, 2007.

CASTIÑEIRA, P. P. Estudo da viabilidade econômica de projetos de recuperação suplementar para campos com alto grau de explotação. Monografia (Bacharelado em Engenharia de Petróleo) - Universidade Federal do Rio de Janeiro, Rio de Janeiro, RJ.

JUNIOR, A. A. S.; CORREIA, J. Revitalização de campos maduros terrestres: uma proposta de regulação. In: $3^{\circ}$ Congresso Brasileiro de P\&D em Petróleo e Gás, 2005, Salvador, BA.

MONTEIRO, N. R. Processo da implantação e consolidação da atividade de produção de petróleo de campos marginais no brasil. In: Produção de Petróleo e Gás em Campos Maduros, 2009, Campinas, SP.
NARVÁEZ, A.; RAMISA, L. R. Reactivation of Mature Fields in Northern Mexico. In: CIPM, 02, 2005, Veracruz, Mexico.

SENNA, B. D. Estudo da viabilidade econômica em campos maduros. 2011. Dissertação (Mestrado em Ciência e Engenharia de Petróleo) - Universidade Federal do Rio Grande do Norte, Natal, RN.

SOLIMAN, M.Y.; EAST, L.; GORELL, S. Reservoir Conformance Approach and Management Practices for Improved Recovery Opportunities: Process and Case History. In: SPE LATIN AMERICAN AND CARIBBEAN PETROLEUM ENGINEERING CONFERENCE, 04, 1999, Caracas, Venezuela. 